\title{
Dihydrotestosterone alters urocortin levels in human umbilical vein endothelial cells
}

\author{
Rong Wan*, Chao Zhu*, Rui Guo, Lai Jin, Yunxin Liu, Li Li, Hao Zhang and Shengnan Li \\ Jiangsu Provincial Key Lab of Cardiovascular Diseases and Molecular Intervention, Department of Pharmacology, \\ Nanjing Medical University, Nanjing 210029, People's Republic of China \\ *(R Wan and C Zhu contributed equally to this work)
}

Correspondence should be addressed

to $\mathrm{S} \mathrm{Li}$

Email

snli@njmu.edu.cn

\begin{abstract}
Urocortin (UCN1) is a member of corticotrophin-releasing factor (CRF) family, which has been proven to participate in inflammation. Previous work showed that dihydrotestosterone (DHT) could promote the inflammatory process. Little is known about the effect of DHT on UCN1 expression. The aim of our study is to investigate the effects and underlying mechanisms of DHT on endothelial UCN1 expression in the absence and presence of induced inflammation. Therefore, we tested the alterations of endothelial UCN1 expression treated with DHT in the presence or absence of lipopolysaccharide (LPS). Our data showed that DHT alone decreased UCN1 levels, which were attenuated in the presence of the androgen receptor (AR) antagonist flutamide. Conversely, in the presence of LPS, DHT augmented the LPS-induced increase in UCN1 expression, which was, interestingly, not affected by flutamide. When cells were treated with DHT alone, AR was upregulated and translocated into the nuclei, which might repress UCN1 expression via a potential androgen-responsive element found in human CRF family promoter. In the presence of LPS, DHT did not influence AR expression and location while it increased toll-like receptor 4 expression and activation, which was not altered by flutamide. DHT enhanced LPS-induced p38MAPK, ERK1/2, and nuclear factor $\kappa$ B pathway activation, which may contribute to the elevated expression of UCN1. These data suggest that DHT differentially influences UCN1 levels under normal and inflammatory conditions in human umbilical vein endothelial cells, which involves AR-dependent and -independent mechanisms respectively.
\end{abstract}

\author{
Key Words \\ - androgen \\ - urocortin \\ - inflammation \\ - lipopolysaccharide
}

\section{Introduction}

Epidemiological and clinical data have indicated that the incidence of cardiovascular diseases is greater in men compared with women of similar age (Vitale et al. 2007). Among women, the incidence of cardiovascular disease is greater in postmenopausal women compared with premenopausal women (Orshal \& Khalil 2004). Despite numerous reports that have suggested the beneficial effects of estrogen replacement therapy (Gerhard \& Ganz 1995), the risk/benefit is still controversial (Enstrom et al. 2002, Grimes \& Lobo 2002). Following the recent reevaluation of the estrogen protection orthodoxy, empirical research has flourished into the role of androgens in the progression of cardiovascular disease (Liu et al. 2003). In addition, clinical data have demonstrated that male hormones are independent factors contributing to the higher male susceptibility to cardiovascular diseases

Published by Bioscientifica Ltd. 
(Ling et al. 2009). It is important to investigate the role of androgens in inflammation owing to the significance of inflammation in the pathogenesis of cardiovascular diseases.

Dihydrotestosterone (DHT), a more potent androgen, is the form of testosterone that undergoes $5 \alpha$-metabolite action (Mareck et al. 2008). DHT has threefold greater affinity than testosterone and 15- to 30-fold greater affinity than adrenal androgens for androgen receptor (AR; Ikeda et al. 2012). Reportedly, DHT enhanced the binding of monocytes to the endothelium via increased expression of vascular cell adhesion molecule-1 in male human endothelial cells (ECs; McCrohon et al. 1999, Death et al. 2004). Furthermore, both in vivo and vitro, DHT treatment was found to enhance nuclear factor $\kappa \mathrm{B}(\mathrm{NF}-\kappa \mathrm{B})$ activity in cerebral arteries and augmented levels of cyclooxygenase- 2 (COX-2) and inducible NO synthase, two well-known pro-inflammatory products of NF- $\kappa \mathrm{B}$ (Gonzales et al. 2009). All these findings suggest that the pro-inflammatory actions of DHT may contribute to sex-related differences in inflammatory diseases.

Situated at the interface between the circulating blood and vasculature, endothelium is not only a protective barrier but also a key player for cardiovascular homeostasis. Particularly, ECs, ingredients of endothelium, are paramount to vascular biology, such as modulating local and systemic inflammation. Importantly, both AR and urocortin (UCN1) have been identified in vascular ECs (Liu et al. 2003, Kageyama et al. 2012). Endothelial UCN1 is upregulated by inflammatory cytokines (Kohno et al. 2001). Our previous studies and others demonstrated that UCN1 could increase EC permeability in the presence of induced inflammation (Cureton et al. 2009, Wan et al. 2013). In addition, UCN1 was reported to augment lipopolysaccharide (LPS)-induced expression of COX-2 and intercellular adhesion molecule 1 in rat aortic ECs by our group (Zhang et al. 2009). Thus, endothelial UCN1 is proven to be involved in the vascular inflammatory process.

There is evidence that a subpopulation of neurons in the PVN expresses both AR and corticotrophin-releasing factor (CRF), which shares $45 \%$ amino acid sequence homology to UCN1. Furthermore, an androgen-responsive element (ARE), which initiated a repressing effect of AR on CRF expression, was identified in the CRF gene promoter region (Bao et al. 2006). Similarly, our in vivo data showed that UCN1 exacerbated the inflammatory process in a rat model of vasculitis induced by sodium laurate (Xu et al. 2009). Furthermore, the inflammatory state was more grave in male rats than in female rats.
Interestingly, pronounced gender-specific differences in UCN1 production were also seen and the gender bias in UCN1 production accounts for the sex difference in vasculitis (original article submitted).

Based on the above results, we hypothesized that DHT may increase UCN1 level and that the role of DHT under normal and inflammatory conditions in induction of endothelial UCN1 expression may be different.

\section{Materials and methods}

\section{Cell culture and reagents}

Human umbilical vein ECs (HUVECs) were obtained from the Institute of Biochemistry (Shanghai, China) and grown in RPMI-1640 medium (Gibco), supplemented with 10\% fetal bovine serum and $1 \%$ penicillin-streptomycin, at $37{ }^{\circ} \mathrm{C}$ in a $95 \% / 5 \% \mathrm{CO}_{2}$ incubator. Our present studies were carried out with HUVECs of passages 5-10.

DHT, AR antagonist flutamide, and LPS were obtained from Sigma. AR antibody was purchased from Signalway Antibody (Pearland, TX, USA). Specific antibody to tolllike receptor 4 (TLR4), NF- $\kappa \mathrm{B}$ p65, and p-NF- $\kappa \mathrm{B}$ p65 were provided by Cell Signaling Technologies (Danvers, MA, USA). UCN1 antibody and HRP-conjugated rabbit antichicken IgG were obtained from Abcam (Cambridge, UK). Antibodies to p38MAPK, p-p38MAPK, ERK1/2, p-ERK1/2, JNK, and p-JNK were from Bioworld Technology (Minnesota, MN, USA). Lipofectamine 2000 transfection reagent, TRIzol, and Moloney murine leukemia virus (MMLV) were from Invitrogen. Cytoplasmic and nuclear protein extraction kit was from Fermentas (Burlington, ON, Canada). Dual-Luciferase Reporter Assay System was obtained from Promega. SYBR green PCR mix was from Bio-Rad. The other reagents were derived from commercial sources.

\section{RNA isolation and PCR}

Total RNAs were extracted from HUVECs using TRIzol reagent according to the manufacturer's protocol. For cDNA synthesis, MMLV was applied as the reverse transcriptase. Quantitative real-time PCR was performed using SYBR green with the ABI Prism 7300 (Applied Biosystems). Primers for UCN1 and $\beta$-actin are listed in Table 1 . The expression levels were calculated based on the $2^{-\Delta \Delta C T}$ methods (Jin et al. 2012) and $\beta$-actin was set as the internal control. For semiquantitative RT-PCR, Tag DNA polymerase was used in the reaction system. Primer sequences for AR (He et al. 2006) and GAPDH

Published by Bioscientifica Ltd 
Table 1 Summary of the qRT-PCR primer sequences used to amplify UCN1 and $\beta$-actin

\begin{tabular}{|c|c|}
\hline & Sequences \\
\hline UCN1 & $\begin{array}{l}\text { Sense: GCTTGCTGGTGAAAAGGACC } \\
\text { Antisense: CTTGCCCACCGAGTCGAAT }\end{array}$ \\
\hline$\beta$-Actin & $\begin{array}{l}\text { Sense: ATGGGTCAGAAGGATTCCTATGT } \\
\text { Antisense: AAGGTCTCAAACATGATCTGGG }\end{array}$ \\
\hline
\end{tabular}

were synthesized from published sequences as shown in Table 2.

\section{Western blot analysis}

The protein of HUVECs was extracted and western blot was performed as described previously (Zhang et al. 2009). Primary antibodies against UCN1 (1:1000), TLR4 (1:1000), p38MAPK (1:1000), p-p38MAPK (1:1000), ERK (1:1000), p-ERK (1:1000), JNK (1:1000), p-JNK (1:1000), p-p65 NF-кB (1:1000), NF-кB (1:10 000), histone (1:5000), GAPDH (1:5000), and $\beta$-actin (1:5000) were used. Immunoreactive proteins were visualized by LumiGLO chemiluminescent reagent and peroxide.

\section{Isolation of nuclear and cytoplasmic proteins}

Nuclear and cytoplasmic proteins of HUVECs were isolated using the cytoplasmic and nuclear protein extraction kit according to the manufacturer's protocol.

\section{Transfection and dual-luciferase reporter assay}

The luciferase reporter construct pGL2-hUS2 containing 2215 nucleotides of hUcn promoter region and 70 nucleotides of the exon 1 (Zhao et al. 1998) was graciously provided by Dr Wylie W Vale (The Clayton Foundation Laboratories for Peptide Biology, The Salk Institute, San Diego, CA, USA). Cells were transfected using Lipofectamine 2000 transfection reagent. Firefly and Renilla luciferase activities were detected separately using the dual-luciferase kit according to the manufacturer's instructions.

\section{Immunofluorescence staining}

Immunofluorescence staining was carried out as described previously (Zhang et al. 2009), with specific antibodies to AR (1:200) and FITC-conjugated anti-rabbit secondary antibody. 4,6-Diamidino-2-phenylindole was used for staining of nuclei. The images were obtained at $20 \times$ magnification using LSM710 confocal microscope (Zeiss, Germany).

\section{Statistical analysis}

Data were expressed as means \pm s.E.M. and analyzed with GraphPad (San Diego, CA, USA) Prism 5 by one-way ANOVA followed by Tukey's test. $P$ values $<0.05$ were considered statistically significant.

\section{Results}

\section{DHT alone decreased UCN1 expression}

To determine the effect of DHT treatment on UCN1 expression in the absence of induced inflammation, a time-course study was performed. HUVECs were treated with DHT $\left(10^{-6} \mathrm{M}\right)$ and harvested at indicated times. As depicted in Fig. 1A and B, DHT reduced UCN1 expression in a time-dependent manner, with the mRNA and protein levels reaching the bottom at 9 and $24 \mathrm{~h}$ respectively. Hence, the time points of 9 and $24 \mathrm{~h}$ were selected in the following studies. Furthermore, DHT decreased the UCN1 expression in a concentration-dependent manner and a marked effect was observed with $10^{-8} \mathrm{M}$ DHT (Fig. 1C and D). Thus, $10^{-8} \mathrm{M}$ DHT was selected as the best given concentration, which is used in the following experiments.

\section{DHT increased the UCN1 expression in the presence of LPS}

To determine the effect of DHT on UCN1 expression under the condition of induced inflammation, HUVECs were treated with LPS $(10 \mu \mathrm{g} / \mathrm{ml})$ in the presence of DHT. As shown in Fig. 2A and B, DHT alone decreased the expression of UCN1, which is consistent with the abovementioned data. However, DHT augmented the LPSincreased UCN1 expression at both mRNA and protein levels. To explore whether AR was involved in the action of DHT, we pretreated the HUVECs with flutamide, the AR antagonist, for $0.5 \mathrm{~h}$ and then DHT and LPS were added in turn. Interestingly, flutamide could abolish the effect of DHT when cells were treated with DHT alone. But when stimulated with LPS, flutamide failed to inhibit the DHT action (Fig. 2C and D). All the data shown suggest that

Table 2 Summary of the RT-PCR primer sequences used to amplify AR and GAPDH

\begin{tabular}{|c|c|c|}
\hline & Sequences & $\begin{array}{l}\text { Product } \\
\text { size }(b p)\end{array}$ \\
\hline$A R$ & $\begin{array}{l}\text { Sense: TCTCAAGAGTTTGGATGGCTCC } \\
\text { Antisense: TCACTGGGTGTGGAAATAGATG }\end{array}$ & 390 \\
\hline GAPDH & $\begin{array}{l}\text { Sense: GGTCGGAGTCAACGGATTTG } \\
\text { Antisense: ATGAGGTCCACCACCCTGTT }\end{array}$ & 969 \\
\hline
\end{tabular}

Published by Bioscientifica Ltd 

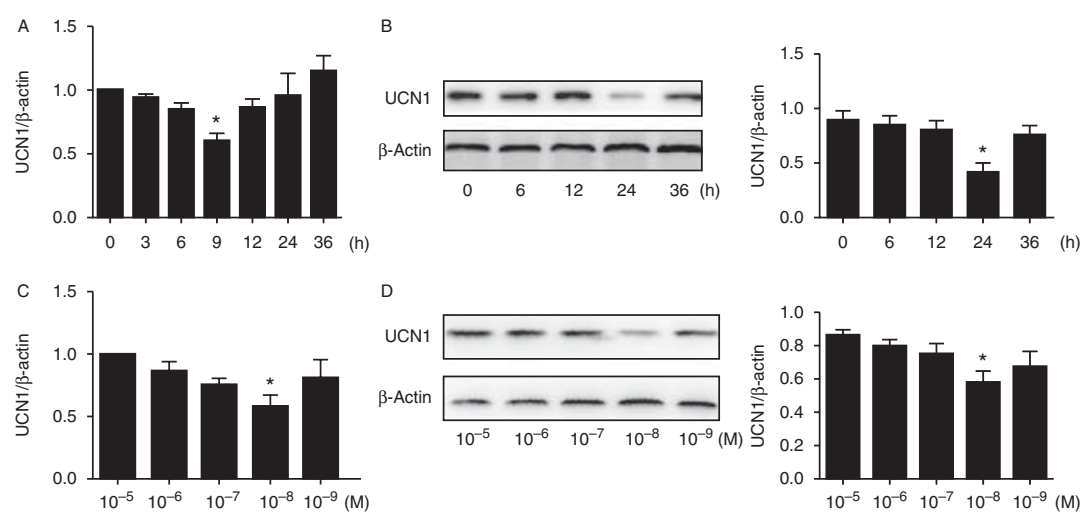

D
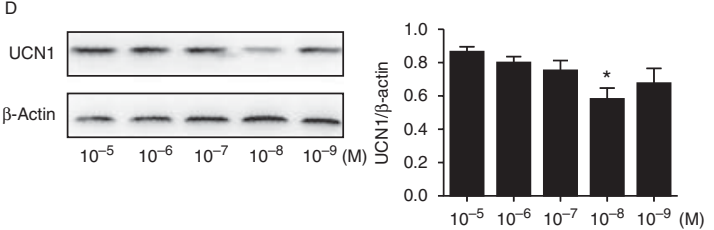

\section{Figure 1}

Treatment with DHT alone altered UCN1 expression in a time- and concentration-dependent manner. (A and B) HUVECs were treated with DHT $\left(10^{-6} \mathrm{M}\right)$ alone and harvested at indicated times. The expression of UCN1 was measured by real-time PCR $(A)$ and western blot (B). DHT decreased UCN1 expression in a time-dependent manner, and the most significant effect of DHT was observed at $9 \mathrm{~h}$ (mRNA) and $24 \mathrm{~h}$ (protein).

DHT decreases the UCN1 expression in an AR-dependent manner and increases the LPS-induced UCN1 expression in an AR-independent manner.

\section{AR repressed UCN1 promoter activity}

To determine whether DHT targeted UCN1 directly, we performed reporter assays. HUVECs were transiently cotransfected with pGL2-hUS2 or pGL2-Basic and treated with vehicle, DHT, LPS, and DHT + LPS. As shown in Fig. 2E, we found that DHT decreased the transcriptional activity significantly while increased the transcriptional activity together with LPS. The effect of DHT on UCN1 promoter activity was inhibited by flutamide. However, flutamide failed to reverse the enlargement action of DHT with LPS together on UCN1 transcriptional activity. Taken together, the above data suggest that DHT could directly regulate the transcriptional action in an AR-dependent manner and that in the presence of LPS, DHT plays as a pro-inflammatory part in an ARindependent manner.

\section{DHT activation altered the AR expression}

Previous data have shown that androgens could induce the upregulation of the levels of AR (Gonzalez-Cadavid et al. 1993). We investigated the AR protein levels after treatment with vehicle or DHT or DHT + LPS by western blot. DHT increased AR protein expression in HUVECs, but in the presence of LPS, AR expression had no alteration compared with vehicle (Fig. 3A).
(C and D) HUVECs were treated with DHT at different concentrations, ranging from $10^{-5}$ to $10^{-9} \mathrm{M}$. The expression of UCN1 was determined by real-time PCR (C) and western blot (D) when cells were treated for 9 and $24 \mathrm{~h}$ respectively. ${ }^{*} P<0.05$. All the data given are the means \pm s.E.M. of values obtained from more than three independent cultures, and a representative experiment is shown.

$\mathrm{AR}$ is a member of the steroid nuclear receptor family, and the transcriptional activity is activated when its nuclear localization is increased (Kemppainen et al. 1992). To further explore the underlying mechanism of AR in UCN1 promoter activity, we first examine the localization of AR treated with vehicle or DHT. As depicted in Fig. 3B, DHT treatment alone increased AR protein expression in both cytoplasm and the nuclei. A similar result was observed by immunofluorescence (Fig. 3C). The expression of AR in the nucleus was significantly increased after treatment with DHT. The above results showed that DHT increased AR nuclear expression.

\section{An ARE was found in UCN1 promoter}

It is well known that ligand-activated AR can regulate gene transcription by binding to specific DNA sequences named ARE in gene promoters (Lundqvist \& Norlin 2012). In order to determine whether AR could act on the UCN1 promoter by an ARE, the human UCN1 promoter region was searched for consensus ARE motifs. The consensus sequence of ARE was determined by Roche et al. (1992). As shown in Fig. 3D, a sequence strongly resembling an ARE was found at the position - 1690. The presence of ARE in UCN1 promoter suggested that AR may repress the UCN1 promoter by ARE.

\section{DHT augmented the TLR4 pathway}

Previous data demonstrated that DHT could increase UCN1 transcription in the presence of LPS. LPS is

Published by Bioscientifica Ltd 

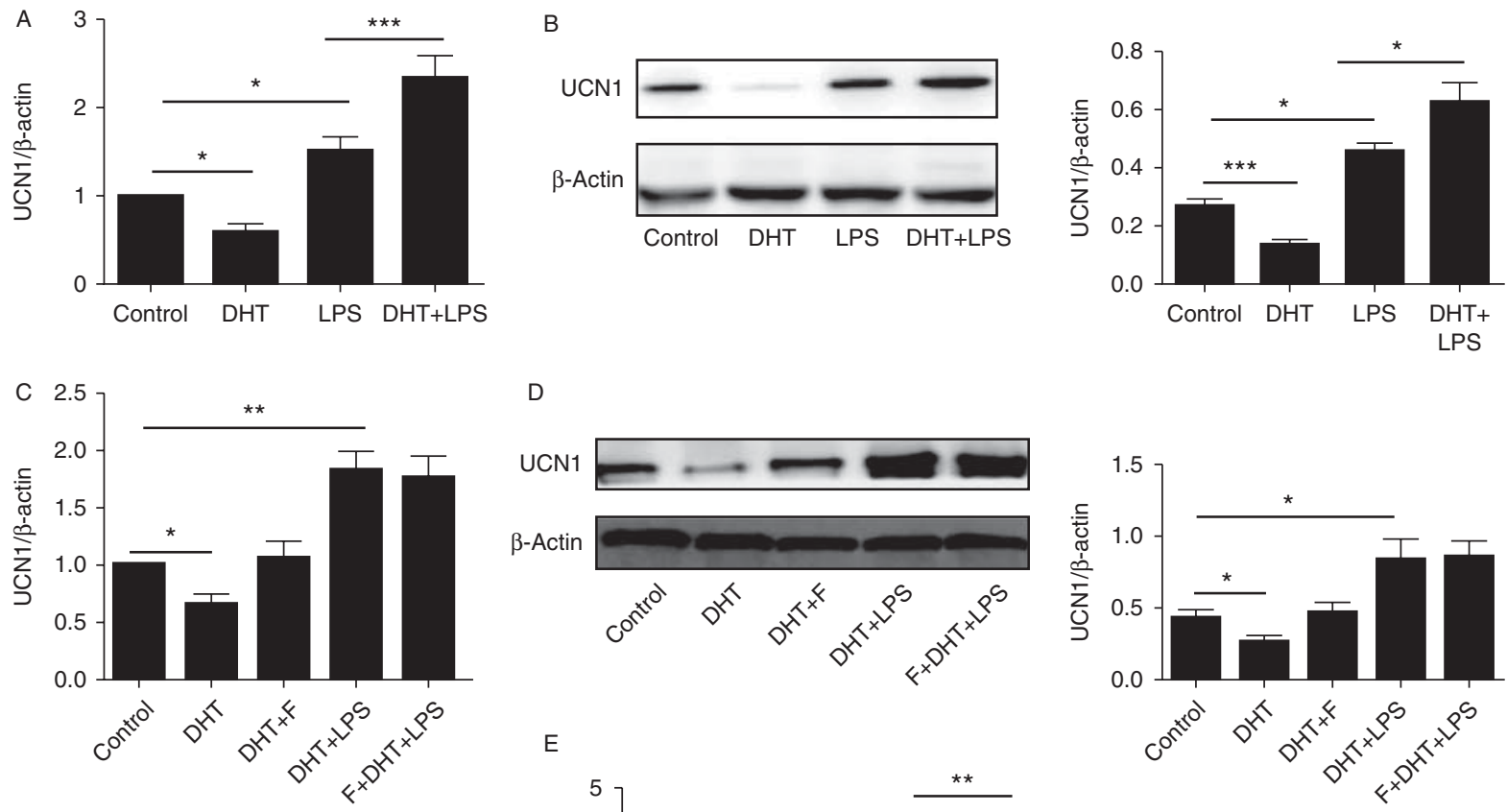

D
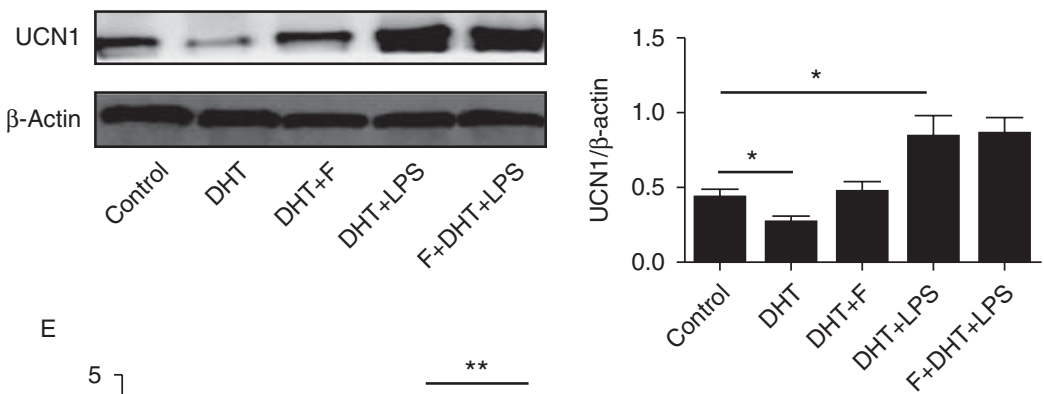

$\mathrm{E}$

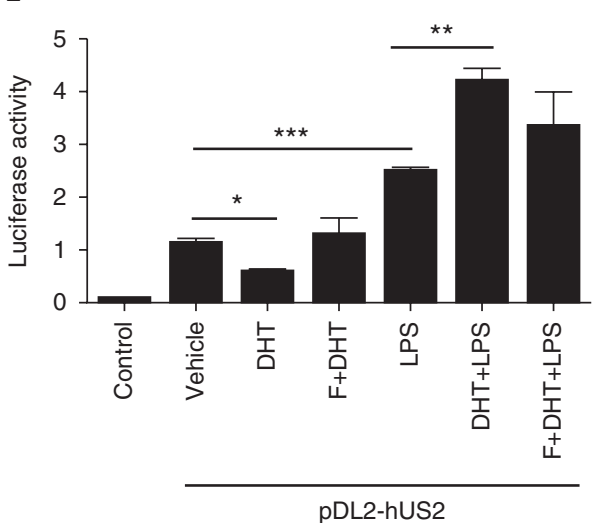

Figure 2

DHT decreased UCN1 expression in an AR-dependent manner but increased UCN1 expression in an AR-independent manner when cells were co-treated with LPS. (A and B) HUVECs were treated with vehicle, DHT $\left(10^{-8} \mathrm{M}\right)$, LPS $(10 \mu \mathrm{g} / \mu \mathrm{l})$, and DHT + LPS for $9 \mathrm{~h}$ (RNA) or $24 \mathrm{~h}$ (protein), and the expression of UCN1 at both mRNA levels and protein levels was determined by real-time PCR ( $A$ ) and western blot (B). (C and D) To determine the involvement of $A R$, flutamide (F), the $A R$ antagonist, was added $0.5 \mathrm{~h}$ before the DHT or DHT + LPS treatment. The UCN1 expressions at both RNA and protein levels were analyzed by real-time PCR (C) and western blot (D). (E) Dual-luciferase reporter assay was performed to further explore the effect of DHT on UCN1 promoter. HUVECs were transfected with pGL2-hUS2 or pGL2-Basic and treated with vehicle, DHT, LPS, and DHT + LPS for $12 \mathrm{~h}$. Luciferase activities were detected. ${ }^{*} P<0.05$, $* * P<0.01$, and $* * * P<0.001$. All the data given are the means \pm s.E.M. of values obtained from more than three independent cultures, and a representative experiment is shown. demonstrated to increase UCN1 expression (Honjo et al. 2006). Thus, it is reasonable to hypothesize that DHT could augment the TLR4 pathway when stimulated by LPS. TLR4 is a member of the TLR superfamily that is required for LPS responsiveness and is involved in the host defense against gram-negative bacteria ( $\mathrm{O}^{\prime} \mathrm{Neill}$ 2000). To explore the mechanisms of DHT in UCN1 transcriptional activity in the presence of LPS, we first measured the expression of TLR4 treated with DHT plus LPS. HUVECs were pretreated with DHT for $0.5 \mathrm{~h}$ and LPS was added. As shown in Fig. 4A, the expression of TLR4 was increased in the presence of DHT together with LPS compared with LPS treatment only.

TLR4-mediated MAPK and NF- $\mathrm{BB}$ signaling plays a key role in the onset of inflammation (Buchanan et al. 2010), which has been implicated in the regulation of UCN1 expression (Brar et al. 1999, Wang et al. 2012). In fact, the activation of MAPK signaling may contribute to the activation of AP-1, another important transcription factor besides NF- $\mathrm{B}$ (Su et al. 2011). To investigate the role of these two pathways in the effect of DHT on LPS-induced UCN1 expression, the phosphorylation 

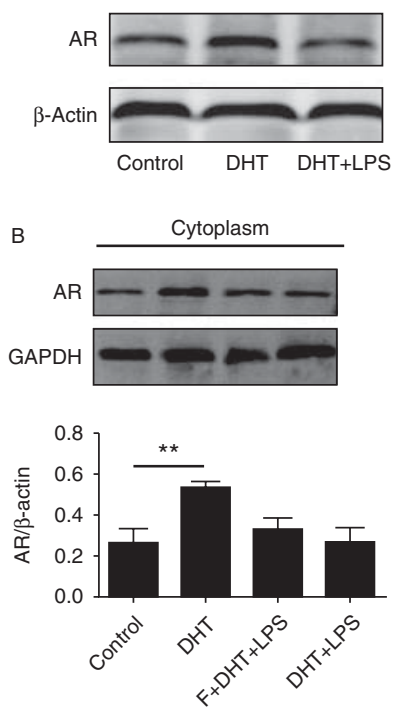
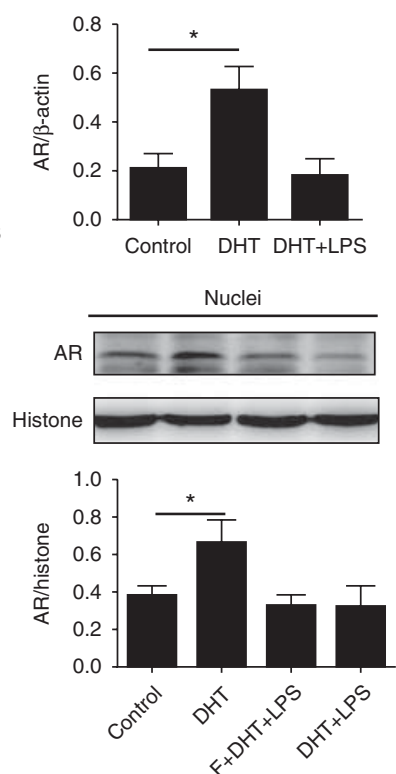
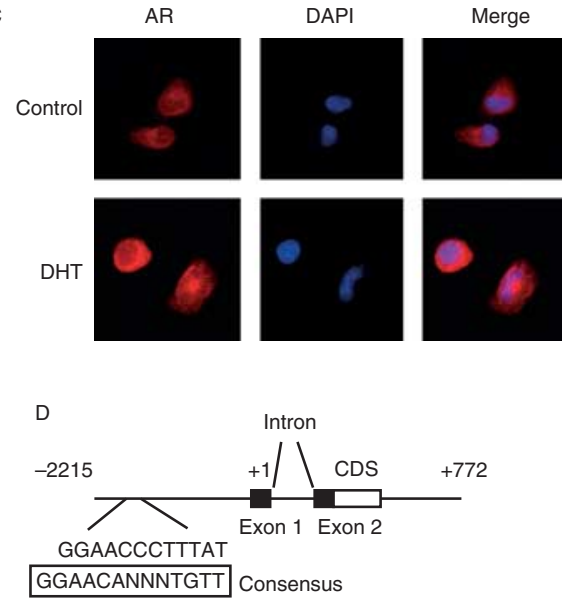

Figure 3

DHT increased AR expression and translocation. (A) HUVECs were treated with $\mathrm{DHT}\left(10^{-8} \mathrm{M}\right)$ for $12 \mathrm{~h}$, and AR expression was determined by western blot. (B) HUVECs were treated as indicated earlier, and the cytoplasmic and nuclear proteins were extracted and subjected to western blot analysis. $* P<0.05$ and $* * P<0.01$. (C) The immunofluorescence images of AR were taken after treatment with DHT for $12 \mathrm{~h}$. All the data given are the means \pm S.E.M. of values obtained from more than three independent cultures, and a representative experiment is shown. (D) Comparison of the putative androgen-responsive element (ARE) in the UCN1 gene promoter region with a consensus $A R E$. Nucleotides relative to the transcriptional start site are shown (AF038633). CDS, coding sequence of UCN1. Exons 1 and 2 of UCN1 gene were indicated. N, any nucleotide. status of NF- $\kappa$ B, p38MAPK, ERK1/2, and JNK was analyzed by western blot.

HUVECs were pretreated with DHT for $0.5 \mathrm{~h}$ and LPS was added subsequently. As depicted in Fig. 4B, DHT induced a significant phosphorylation of p38MAPK and ERK1/2 with peak activation at $2 \mathrm{~h}$ in the presence of LPS. Consistent with the results observed in UCN1 expression, this DHT effect was independent of AR (Fig. $4 \mathrm{C})$. However, DHT failed to alter the phosphorylation of JNK (Fig. 4B).

Next, we examined the effect of DHT on the NF- $\kappa$ B pathway. First, the protein levels of NF- $\kappa \mathrm{B}$ were determined in nuclear fractions. The NF- $\kappa \mathrm{B}$ expression was increased in nuclear fractions after $2 \mathrm{~h}$ of treatment with LPS in the presence of DHT (treatment for $0.5 \mathrm{~h}$ before LPS application; Fig. 4D). Next, the phosphorylation status of NF- $\kappa \mathrm{B}$ was determined by western blot. As shown in Fig. 4E, LPS treatment increased the phosphorylation of $\mathrm{NF}-\kappa \mathrm{B}$, and DHT application significantly enhanced it. Taken together, we came to the conclusion that DHT could increase LPS-induced phosphorylation of p38MAPK, ERK1/2, and NF- $\mathrm{B}$, which may contribute to the elevated level of UCN1.

\section{Discussion and conclusions}

The aim of this study was to explore the effects and mechanisms of androgens on UCN1 expression in ECs. We examined the effects of nonaromatizable androgen DHT on UCN1 expression in the absence or presence of LPS. In that, both AR and UCN1 express in HUVECs, and the cell line of HUVECs was chosen for the research. In this study, we focused on DHT actions on UCN1 expression in ECs. Hence, the obtained data can only display the action features of DHT in HUVECs. More experiments in adult ECs are needed for adult disease-related aspects. Our data indicated that DHT alone could significantly decrease UCN1 expression at both mRNA and protein levels in an AR-dependent manner. On the contrary, in the presence of LPS, DHT increased UCN1 expression in an AR-independent manner as the AR antagonist, flutamide, did not influence this effect of DHT. TLR4 expression was elevated and TLR4-mediated NF- $\kappa$ B and MAPK pathways were activated. Thus, we conclude that DHT differentially influences UCN1 levels under normal and inflammatory conditions in HUVECs, in which AR-dependent and -independent mechanisms were involved respectively (Fig. 5).

Published by Bioscientifica Ltd. 
A

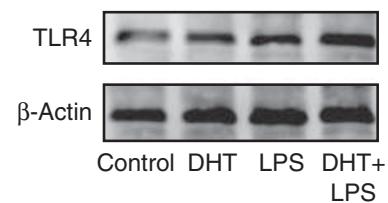

B

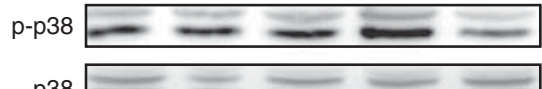

p38

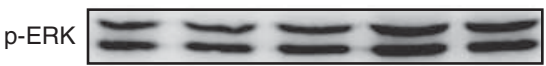

ERK
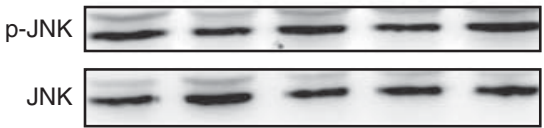

$\beta$-Actin

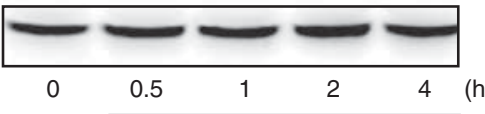

DHT+LPS

D

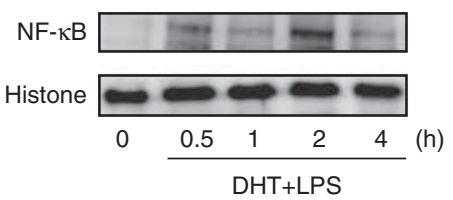

Figure 4

DHT enhanced the TLR4 pathway under an inflammatory condition. (A) HUVECs were treated with vehicle, DHT, LPS, or DHT + LPS for $24 \mathrm{~h}$, and the expression of TLR4 was examined by western blot. (B) HUVECs were treated with DHT plus LPS and harvested at indicated times. The phosphorylation levels of MAPKs (p38MAPK, p-ERK1/2, and JNK) were determined by western blot. (C) To explore the involvement of AR, flutamide was added. After treatment with vehicle, LPS, DHT+LPS, or

UCN1 is reported to be upregulated under inflammatory conditions. In our experimental animal model, a significantly increased expression of UCN1 mRNA was found in the asthma group ( $\mathrm{Wu}$ et al. 2006). Other investigators have shown similar findings in inflammatory diseases such as arthritis (Kohno et al. 2001), hypertension (Florio et al. 2006), and atherosclerosis (Inada et al. 2009). UCN1 is demonstrated to promote the development of vasculitis in vivo by our group (Xu et al. 2009). Moreover, UCN1-positive cells were found to be correlated with the severity of inflammation in colonic mucosa with ulcerative colitis (Saruta et al. 2004). Our observations taken together with those of other's suggest that UCN1 is an important factor in inflammation.
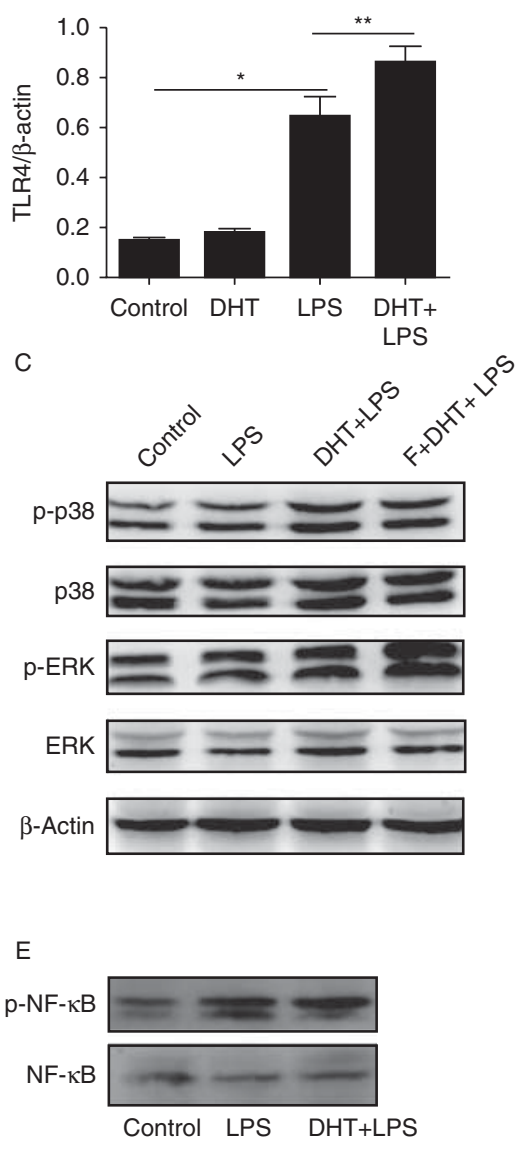

flutamide $(F)+D H T+$ LPS for $2 \mathrm{~h}$, the phosphorylation levels of p38MAPK and ERK $1 / 2$ were determined. (D) The phosphorylation levels of NF- $\kappa B$ treated at indicated times were determined by western blot. (E) HUVECs were treated with vehicle, LPS, or DHT + LPS for $2 \mathrm{~h}$; the phosphorylation levels of NF- $\kappa \mathrm{B}$ were determined by western blot. ${ }^{*} P<0.05$ and $* * P<0.01$. All the data given are the means \pm s.E.M. of values obtained from more than three independent cultures and a representative experiment is shown.

Testosterone, the major androgen in circulation, is responsible for androgenic effects, but its role in cardiovascular diseases is still controversial. A key factor that can account for the discrepancies of testosterone actions is whether testosterone exerts effects either directly on AR or converts to the potent androgen, DHT, or aromatization to estrogen (Gonzales et al. 2009). DHT is considered to be a clear marker of androgen action rather than testosterone. DHT failed to convert into estrogen by aromatase, which makes the results not subject to the confounding potential interpretations of ER- and AR-mediated effects (Death et al. 2004). Our in vivo data showed that, in a laurate-induced rat's peripheral arterial vasculitis model, the inflammatory status was much more serious in male rats than in female

Published by Bioscientifica Ltd 

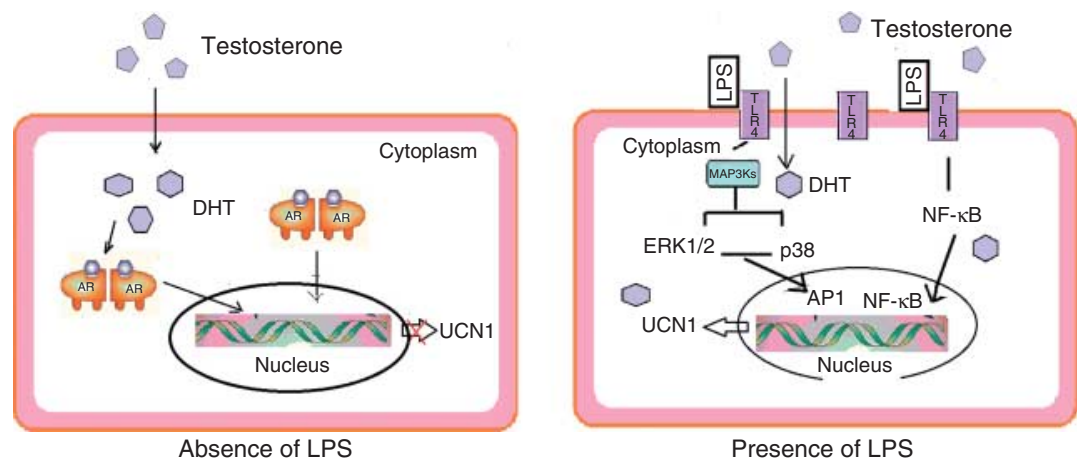

Figure 5

Model for DHT-medicated pathway in endothelial UCN1 expression under normal and inflammatory conditions.

rats (unpublished data). Furthermore, in male rats, the serum UCN1 level is higher than that in female rats. All the data suggest that UCN1 may be a key factor in gender difference of vascular inflammation. Thus, to investigate the association between DHT and UCN1 is important.

Consistent with our in vivo data, DHT increased UCN1 expression in the presence of LPS. Interestingly, under normal conditions, DHT repressed UCN1 expression. It was of interest to find that there was a specific ARE present in the human UCN1 promoter region. Similarly, Bao et al. (2006) showed a potential ARE in the human CRF promoter and testosterone repressed CRF promoter activity through the ARE. Thus, it appears that, in the absence of an inflammatory stimulus, DHT acts via the classical AR to repress UCN1 expression, but in the presence of LPS, DHT performs via an AR-independent mechanism to increase UCN1 levels. Our results are in accordance with the report that DHT differentially influences COX-2 levels under physiological and pathophysiological conditions in human coronary artery smooth muscle cells (Osterlund et al. 2010).

Our results first demonstrated that DHT increased LPSinduced TLR4 expression and augmented the TLR4mediated signaling activation in an AR-independent manner. MAPK kinases are a group of highly conserved serine/threonine protein kinases that are involved in LPS-TLR4-medicated cytokine expression, including UCN1 (Wang et al. 2012). In human placenta, the MAPK signal transduction pathway played a pivotal role in the regulation of CRF gene expression (Cheng \& Handwerger 2005). At least three distinctly regulated groups of MAPK have been identified, ERK1/2, JNK, and p38MAPK (Krishna \& Narang 2008). It is well known that p38MAPK ameliorated the stabilization of ARE-containing mRNAs in posttranscriptional regulation (Kaminska 2005). In addition, activated JNK and ERK1/2 are reported to increase AP-1 activity (Rahman \& MacNee 1998, Uto et al. 2005), and the promoter sequence of CRH contains an AP-1 site (Behan et al. 1993). Consistent with others, in this study, we found that ERK1/2 and p38MAPK were activated by DHT + LPS, but JNK was not affected. These results highly suggest that DHT increased UCN1 expression by augmenting the LPS-induced MAPK activation. NF- $\mathrm{KB}$ is another important nuclear transcription factor that is mediated by TLR4 stimulation (Jiang et al. 2000). Recently, $\mathrm{NF}-\kappa \mathrm{B}$ was shown to be involved in the regulation of UCN2 mRNA, another member of CRF family (Ikeda et al. 2009). Importantly, CRF promoter contains multiple NF-кB binding sites (Kageyama et al. 2010). Our present work demonstrated that DHT could promote the activation of NF- $\mathrm{B}$ stimulated by LPS. Taken together with the above reports, DHT increased UCN1 expression in the presence of LPS and the mechanism may involve NF-кB signaling.

In conclusion, we here demonstrate that DHT alone decreases endothelial UCN1 expression via AR while increases endothelial UCN1 expression in the presence of LPS. Under normal conditions, DHT may decrease the UCN1 promoter through ARE. Under the circumstance of induced inflammation, DHT increased endothelial UCN1 and augmented LPS-TLR4 signaling.

\section{Declaration of interest}

The authors declare that there is no conflict of interest that could be perceived as prejudicing the impartiality of the research reported.

\section{Funding}

This work was supported by the Natural Science Foundation of China (nos 81273510 and 81072668), Jiangsu Provincial Funds (nos BK2010078 and 10KJA350031), and Priority Academic Program Development of Jiangsu Higher Education Institutions.

Published by Bioscientifica Ltd. 


\section{References}

Bao AM, Fischer DF, Wu YH, Hol EM, Balesar R, Unmehopa UA, Zhou JN \& Swaab DF 2006 A direct androgenic involvement in the expression of human corticotropin-releasing hormone. Molecular Psychiatry 11 567-576. (doi:10.1038/sj.mp.4001800)

Behan DP, Potter E, Lewis KA, Jenkins NA, Copeland N, Lowry PJ \& Vale WW 1993 Cloning and structure of the human corticotrophin releasing factor-binding protein gene (CRHBP). Genomics 16 63-68. (doi:10.1006/ geno.1993.1141)

Brar BK, Stephanou A, Okosi A, Lawrence KM, Knight RA, Marber MS \& Latchman DS 1999 CRH-like peptides protect cardiac myocytes from lethal ischaemic injury. Molecular and Cellular Endocrinology 158 55-63. (doi:10.1016/S0303-7207(99)00183-5)

Buchanan MM, Hutchinson M, Watkins LR \& Yin H 2010 Toll-like receptor 4 in CNS pathologies. Journal of Neurochemistry 114 13-27. (doi:10.1111/j.1471-4159.2010.06736.x)

Cheng YH \& Handwerger S 2005 Mitogen-activated protein kinase activation induces corticotrophin-releasing hormone gene expression in human placenta. Life Sciences 77 1263-1272. (doi:10.1016/j.lfs.2005. 03.009)

Cureton EL, Ereso AQ, Victorino GP, Curran B, Poole DP, Liao M, Harken AH \& Bhargava A 2009 Local secretion of urocortin 1 promotes microvascular permeability during lipopolysaccharide-induced inflammation. Endocrinology 150 5428-5437. (doi:10.1210/en.2009-0489)

Death AK, McGrath KC, Sader MA, Nakhla S, Jessup W, Handelsman DJ \& Celermajer DS 2004 Dihydrotestosterone promotes vascular cell adhesion molecule-1 expression in male human endothelial cells via a nuclear factor-кB-dependent pathway. Endocrinology 145 1889-1897. (doi:10.1210/en.2003-0789)

Enstrom I, Lidfeldt J, Lindholm LH, Nerbrand C, Pennert K \& Samsioe G 2002 Does blood pressure differ between users and non-users of hormone replacement therapy? The Women's Health In the Lund Area (WHILA) Study Blood Pressure 11 240-243. (doi:10.1080/ 08037050213757)

FlorioP, Torricelli M, DeFalco G, Leucci E, Giovannelli A, GazzoloD, Severi FM, Bagnoli F, Leoncini L, Linton EA etal. 2006 High maternal and fetal plasma urocortin levels in pregnancies complicated by hypertension. Journal of Hypertension 24 1831-1840. (doi:10.1097/01.hjh.0000242408.65783.b2)

Gerhard M \& Ganz P 1995 How do we explain the clinical benefits of estrogen? From bedside to bench Circulation 92 5-8. (doi:10.1161/ 01.CIR.92.1.5)

Gonzales RJ, Duckles SP \& Krause DN 2009 Dihydrotestosterone stimulates cerebrovascular inflammation through $\mathrm{NF \kappa B}$, modulating contractile function. Journal of Cerebral Blood Flow and Metabolism 29 244-253. (doi:10.1038/jcbfm.2008.115)

Gonzalez-Cadavid N, Vernet D, Fuentes Navarro A, RodriguezJA, Swerdloff RS \& Rajfer J 1993 Up-regulation of the levels of androgen receptor and its mRNA by androgens in smooth-muscle cells from rat penis. Molecular and Cellular Endocrinology 90 219-229. (doi:10.1016/0303-7207(93)90155-D)

Grimes DA \& Lobo RA 2002 Perspectives on the Women's Health Initiative trial of hormone replacement therapy. Obstetrics and Gynecology 100 1344-1353. (doi:10.1016/S0029-7844(02)02503-6)

He ML, Yuan HQ, Jiang AL, Gong AY, Chen WW, Zhang PJ, Young CY \& Zhang JY 2006 Gum mastic inhibits the expression and function of the androgen receptor in prostate cancer cells. Cancer 106 2547-2555. (doi:10.1002/cncr.21935)

Honjo T, Inoue N, Shiraki R, Kobayashi S, Otsui K, Takahashi M, Hirata K, Kawashima S, Yokozaki H \& Yokoyama M 2006 Endothelial urocortin has potent antioxidative properties and is upregulated by inflammatory cytokines and pitavastatin. Journal of Vascular Research 43 131-138. (doi:10.1159/000090132)

Ikeda K, Tojo K, Inada Y, Takada Y, Sakamoto M, Lam M, Claycomb WC \& Tajima N 2009 Regulation of urocortin I and its related peptide urocortin II by inflammatory and oxidative stresses in HL-1 cardiomyocytes. Journal of Molecular Endocrinology 42 479-489. (doi:10.1677/JME-08-0151)

Ikeda Y, Aihara K, Yoshida S, Akaike M \& Matsumoto T 2012 Effects of androgens on cardiovascular remodeling. Journal of Endocrinology $\mathbf{2 1 4}$ 1-10. (doi:10.1530/JOE-12-0126)

Inada Y, Ikeda K, Tojo K, Sakamoto M, Takada Y \& Tajima N 2009 Possible involvement of corticotropin-releasing factor receptor signaling on vascular inflammation. Peptides 30 365-372. (doi:10.1016/j.peptides. 2008.10.015)

Jiang Q, Akashi S, Miyake K \& Petty HR 2000 Lipopolysaccharide induces physical proximity between CD14 and toll-like receptor 4 (TLR4) prior to nuclear translocation of NF-к B. Journal of Immunology 165 3541-3544.

Jin L, Chen C, Guo R, Wan R \& Li S 2012 Role of corticotropin-releasing hormone family peptides in androgen receptor and vitamin $\mathrm{D}$ receptor expression and translocation in human breast cancer MCF-7 cells. European Journal of Pharmacology 684 27-35. (doi:10.1016/j.ejphar. 2012.03.034)

Kageyama K, Kagaya S, Takayasu S, Hanada K, Iwasaki Y \& Suda T 2010 Cytokines induce NF- $\mathrm{kB}$, Nurr1 and corticotropin-releasing factor gene transcription in hypothalamic 4B cells. Neuroimmunomodulation $\mathbf{1 7}$ 305-313. (doi:10.1159/000292022)

Kageyama K, Teui K, Tamasawa N \& Suda T 2012 Regulation and roles of urocortins in the vascular system. International Journal of Endocrinology 2012 873723. (doi:10.1155/2012/873723)

Kaminska B 2005 MAPK signalling pathways as molecular targets for antiinflammatory therapy - from molecular mechanisms to therapeutic benefits. Biochimica et Biophysica Acta 1754 253-262. (doi:10.1016/j. bbapap.2005.08.017)

Kemppainen JA, Lane MV, Sar M \& Wilson EM 1992 Androgen receptor phosphorylation, turnover, nuclear transport, and transcriptional activation. Specificity for steroids and antihormones. Journal of Biological Chemistry 267 968-974.

Kohno M, Kawahito Y, Tsubouchi Y, Hashiramoto A, Yamada R, Inoue KI, Kusaka Y, Kubo T, Elenkov IJ, Chrousos GP et al. 2001 Urocortin expression in synovium of patients with rheumatoid arthritis and osteoarthritis: relation to inflammatory activity. Journal of Clinical Endocrinology and Metabolism 86 4344-4352. (doi:10.1210/jc.86.9.4344)

Krishna M \& Narang H 2008 The complexity of mitogen-activated protein kinases (MAPKs) made simple. Cellular and Molecular Life Sciences 65 3525-3544. (doi:10.1007/s00018-008-8170-7)

Ling S, Komesaroff PA \& Sudhir K 2009 Cardiovascular physiology of androgens and androgen testosterone therapy in postmenopausal women. Endocrine, Metabolic \& Immune Disorders Drug Targets 9 29-37. (doi:10.2174/187153009787582414)

Liu PY, Death AK \& Handelsman DJ 2003 Androgens and cardiovascular disease. Endocrine Reviews 24 313-340. (doi:10.1210/er.2003-0005)

Lundqvist J \& Norlin M 2012 Effects of CYP7B1-related steroids on androgen receptor activation in different cell lines. Biochimica et Biophysica Acta 1821 973-979. (doi:10.1016/j.bbalip.2012.03.007)

Mareck U, Geyer H, Opfermann G, Thevis M \& Schanzer W 2008 Factors influencing the steroid profile in doping control analysis. Journal of Mass Spectrometry 43 877-891. (doi:10.1002/jms.1457)

McCrohon JA, Jessup W, Handelsman DJ \& Celermajer DS 1999 Androgen exposure increases human monocyte adhesion to vascular endothelium and endothelial cell expression of vascular cell adhesion molecule-1. Circulation 99 2317-2322. (doi:10.1161/01.CIR.99.17.2317)

O'Neill L 2000 The toll/interleukin-1 receptor domain: a molecular switch for inflammation and host defence. Biochemical Society Transactions 28 557-563.

Orshal JM \& Khalil RA 2004 Gender, sex hormones, and vascular tone. American Journal of Physiology. Regulatory, Integrative and Comparative Physiology 286 R233-R249. (doi:10.1152/ajpregu.00338.2003)

Osterlund KL, Handa RJ \& Gonzales RJ 2010 Dihydrotestosterone alters cyclooxygenase-2 levels in human coronary artery smooth muscle

Published by Bioscientifica Ltd. 
cells. American Journal of Physiology. Endocrinology and Metabolism 298 E838-E845. (doi:10.1152/ajpendo.00693.2009)

Rahman I \& MacNee W 1998 Role of transcription factors in inflammatory lung diseases. Thorax 53 601-612. (doi:10.1136/thx.53.7.601)

Roche PJ, Hoare SA \& Parker MG 1992 A consensus DNA-binding site for the androgen receptor. Molecular Endocrinology 6 2229-2235. (doi:10.1210/me.6.12.2229)

Saruta M, Takahashi K, Suzuki T, Torii A, Kawakami M \& Sasano H 2004 Urocortin 1 in colonic mucosa in patients with ulcerative colitis. Journal of Clinical Endocrinology and Metabolism 89 5352-5361. (doi:10.1210/jc.2004-0195)

Su YW, Chiou WF, Chao SH, Lee MH, Chen CC \& Tsai YC 2011 Ligustilide prevents LPS-induced iNOS expression in RAW 264.7 macrophages by preventing ROS production and down-regulating the MAPK, NF- $\mathrm{B}$ and AP-1 signaling pathways. International Immunopharmacology $\mathbf{1 1}$ 1166-1172. (doi:10.1016/j.intimp.2011.03.014)

Uto T, Fujii M \& Hou DX 2005 6-(Methylsulfinyl)hexyl, isothiocyanate suppresses inducible nitric oxide synthase expression through the inhibition of janus kinase 2-mediated JNK pathway in lipopolysaccharide-activated murine macrophages. Biochemical Pharmacology 70 1211-1221. (doi:10.1016/j.bcp.2005.07.011)

Vitale C, Miceli M \& Rosano GM 2007 Gender-specific characteristics of atherosclerosis in menopausal women: risk factors, clinical course and strategies for prevention. Climacteric 10(Suppl 2) 16-20. (doi:10.1080/ 13697130701602712)
Wan R, Guo R, Chen C, Jin L, Zhu C, Zhang Q, Xu Y \& Li S 2013 Urocortin increased LPS-induced endothelial permeability by regulating the cadherin-catenin complex via corticotrophin-releasing hormone receptor 2. Journal of Cellular Physiology 228 1295-1303. (doi:10.1002/ jcp.24286)

Wang W, Zhang X, Yang L, Liu D, Liu G \& Zhou J 2012 Lipopolysaccharide upregulates the expression of corticotropin-releasing hormone via MAP kinase pathway in rat peritoneal macrophages. Molecular and Cellular Biochemistry 361 1-7. (doi:10.1007/s11010-011-1080-2)

Wu Y, Zhou H, Xu Y \& Li S 2006 Enhanced expression of urocortin in lung tissues of rats with allergic asthma. Biochemical and Biophysical Research Communications 341 532-540. (doi:10.1016/j.bbrc.2005.12.214)

Xu Y, Zhang R, Chen J, Zhang Q, Wang J, Hu J, Guan X, Jin L, Fu H, Gui B et al. 2009 Urocortin promotes the development of vasculitis in a rat model of thromboangiitis obliterans via corticotrophin-releasing factor type 1 receptors. British Journal of Pharmacology 157 1368-1379. (doi:10. 1111/j.1476-5381.2009.00210.x)

Zhang R, Xu Y, Fu H, Wang J, Jin L \& Li S 2009 Urocortin induced expression of COX-2 and ICAM-1 via corticotrophin-releasing factor type 2 receptor in rat aortic endothelial cells. British Journal of Pharmacology 158 819-829. (doi:10.1111/j.1476-5381.2009.00346.x)

Zhao L, Donaldson CJ, Smith GW \& Vale WW 1998 The structures of the mouse and human urocortin genes (Ucn and UCN). Genomics $5023-33$. (doi:10.1006/geno.1998.5292)

Received in final form 19 May 2013

Accepted 24 June 2013

Accepted Preprint published online 24 June 2013
(C) 2013 Society for Endocrinology Printed in Great Britain 